\title{
Effects of Earth's curvature and radial hetero- geneity in dislocation studies: Case studies of the 2008 Wenchuan earthquake and the 2004 Sumatra earthquake*
}

\author{
Guangyu Fu ${ }^{1,2, \uparrow}$ Wenke Sun ${ }^{3}$ Yoichi Fukuda ${ }^{2}$ \\ Shanghua Gao ${ }^{1}$ and Takashi Hasegawa ${ }^{2}$ \\ ${ }^{1}$ Institute of Earthquake Science, China Earthquake Administration, Beijing 100036, China \\ ${ }^{2}$ Department of Geophysics, Graduate School of Science, Kyoto University, Kyoto 606-8502, Japan \\ ${ }^{3}$ Laboratory of Computational Geodynamics, Graduate University of Chinese Academy of Sciences, \\ Beijing 100049, China
}

\begin{abstract}
Recently, effects of Earth's curvature and radial heterogeneity on coseismic deformations are often investigated based on the 2004 Sumatra earthquake. However, such effects are strongly related to earthquake types. As a low dip angle event, the 2004 Sumatra earthquake is not a good seismic case for such a topic since the effects for moderate dip angle events are much bigger. In this study, the half-space and spherical dislocation theories are used, respectively, to calculate coseismic displacements caused by the 2008 Wenchuan earthquake and the 2004 Sumatra earthquake. Effects of Earth's curvature and stratification are investigated through the discrepancies of results calculated using the two dislocation theories. Results show that the effects of Earth's curvature and stratification for the 2008 Wenchuan earthquake are much larger than those for the 2004 Sumatra earthquake. Ignoring the effects will cause errors up to $100 \%-200 \%$ in far field displacements for a moderate dip angle event like the 2008 Wenchuan earthquake. Such great effects are much bigger than those conclusions of previous studies. Besides, comparison with observations verifies that spherical dislocation theories yield better results than half-space ones in far fields.
\end{abstract}

Key words: coseismic displacement; dislocation theory; Earth model

CLC number: P315.2 Document code: A

\section{Introduction}

We can interpret geodetic deformations such as displacement, tilt, strain, and gravity change caused by earthquakes or volcanoes using dislocation theories because earthquakes and volcanic events can be modeled by fault movement (dislocation) within the Earth. Since Steketee (1958) introduced dislocation theory into the seismic study field, dislocation theories have been presented for different media model types such as a homogeneous half-space Earth model (e.g. Okada, 1985); a spherically symmetric, non-rotating, perfectly elastic

\footnotetext{
* Received 28 January 2009; accepted in revised form 31 May 2009; published 10 August 2010.

* Corresponding author. e-mail: fugygao@gmail.com

(C) The Seismological Society of China and Springer-Verlag Berlin Heidelberg 2010
}

and isotropic (SNREI) Earth model (e.g. Sun and Okubo, 1993); a viscoelastic spherical Earth model (e.g. Tanaka et al, 2006); a 3D heterogeneous spherical Earth model (e.g. Fu and Sun, 2008); etc. Among them, dislocation theories for a homogeneous half-space earth model (e.g., Okada, 1985; Okubo, 1991; 1992) are most widely used to compute coseismic deformations because of their mathematical simplicity. However, because the actual Earth more closely approximates an inhomogeneous sphere than a homogeneous half-space, Earth's spherical curvature and stratification might engender large errors when half-space theories are used to analyze far-field deformations. A theoretical investigation is therefore desirable to clarify the effects of Earth's sphericity and stratified structure.

Sabadini and Vermeersen (1997) investigated the in- 
fluence of lithospheric and mantle stratification on global post-seismic deformation based on the normal mode technique. They found that the mantle viscosity structure strongly influenced post-seismic deformation in a far field. However, their discussion was limited to a dip-slip source. Sun and Okubo (2002) studied the effect of Earth's spherical curvature and radial heterogeneity by comparing the Green functions of different dislocation theories. Their results showed very large effects of sphericity and stratification. The effect of a layered structure reaches a discrepancy of more than $25 \%$ everywhere on the Earth's surface, including the near field. However, their discussion was limited to simple point dislocations. To date, such investigations for actual seismic events are always based on the 2004 Sumatra earthquake (Banerjee et al, 2005, 2007; Sun et al, 2009). However, the effects of Earth's curvature and radial heterogeneities are related strongly to earthquake types. This study will show that the 2004 Sumatra earthquake is not a good seismic case for investigation the effects of Earth's curvature and heterogeneity on coseismic deformations therefore the estimations of previous studies (Banerjee et al, 2005, 2007; Sun et al, 2009) are too small.

This paper presents effects of Earth's curvature and stratification on coseismic displacements caused respectively by the 2008 Wenchuan earthquake and $2004 \mathrm{Su}-$ matra earthquake, respectively. The distribution patterns around the epicenter can yield direct images of those effects. Results show that the effects of Earth's curvature and stratification are very large, but they vary for different seismic types. For moderate dip angle events such as the 2008 Wenchuan earthquake, ignoring the effects may cause $100 \%-200 \%$ errors for coseismic displacements in far fields. The greater the epicentral distance is, the greater the effects will be. Therefore, we recommend spherical dislocation theories instead of half-space ones to interpret the coseismic deformations in far fields or to invert seismic fault models using far-field geodetic data.

\section{Comparison between coseismic dis- placements calculated by half-space and spherical dislocation theories: Effects of Earth's curvature and stratification}

Scientists often use dislocation theories to interpret Earth's responses to earthquakes or volcanoes (Maruyama, 1964; Okada, 1985; Sun et al, 1996; Pollitz, 2003; etc.). In those studies, Earth is often simplified as some simple geometric model such as a homogeneous half-space, a layered sphere, a 3D heterogeneous sphere, etc. The homogeneous half-space Earth model is the simplest; it assumes the Earth to be a half-space filled with homogeneous elastic medium (Figure 1a). For such a simple Earth model, it is easy to express the seismic response of the medium mathematically. For example, Okada (1985) presented a complete set of analytical formulae for calculating coseismic displacements, tilts, and strain changes caused by shear and tensile dislocations. Okubo $(1991 ; 1992)$ proposed expressions in closed form to describe potential and gravity changes caused by dislocations. Because of their mathematical simplicity, these dislocation theories have been widely applied to studying Earth's responses to earthquakes or volcanoes or to inverting seismic faults. However, the validity of these theories is limited to near fields because the Earth's curvature and radial heterogeneity are not considered at all. Model geodesy is able to detect far field deformations. Dislocation theories for a more realistic Earth model are therefore necessary to interpret far field deformations. Under this background, Sun and Okubo (1993; 1998), Sun et al (1996, 2006) presented dislocation theories based on a radial inhomogeneous spherical Earth model (Figure 1b). These theories are valid for the entire Earth surface because they include Earth's sphericity and stratification.

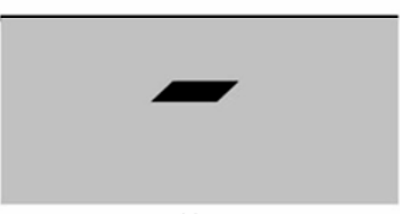

(a)

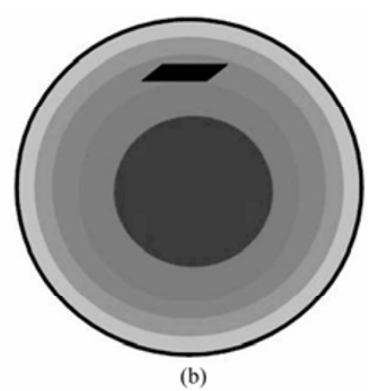

Figure 1 Two Earth models used for dislocation studies. (a) A homogeneous half-space Earth model; (b) A layered spherical Earth model.

This study investigates effects of Earth's curvature and stratification through discrepancies between the results calculated by half-space and spherical dislocation theories. For simplifying discussions, and without losing generality, we limit our concentration to displacements caused by earthquakes. Discussions for the other geophysical deformations such as tilt, strain, and gravity change would be similar. We also constrain our discussion to coseismic deformations so that the corresponding half-space dislocation theory of Okada (HDTO) and 
spherical dislocation theory of Sun et al (SDTS) are directly useful. In this study, a layered spherical Earth model named PREM (Dziewonski and Anderson, 1981) is used for the spherical dislocation theory.

To calculate coseismic displacements caused by earthquakes with large geometrical size of faults such as the 2008 Wenchuan earthquake and the 2004 Sumatra earthquake, the segment-summation scheme proposed by Fu and Sun (2004) is necessary. Conceptually, it divides the large fault plane into limited sub-faults so that the coseismic displacements caused by each sub-fault can be evaluated by direct application of HDTO or SDTS and by subsequently summing up the individual contributions from all sub-faults. Each sub-fault is sufficiently small to be regarded as having a homogeneous dislocation movement, which is always possible because the source rupture process in seismology always provides such a fault model.

If we try to judge which dislocation theory (SDTS or HDTO) is better by comparing theoretical calculations with GPS observations, we must choose a suitable fault model for our calculation. We should avoid using those fault models which are inverted by GPS data because the half-space dislocation theories such as HDTO are always used during the inversion; therefore, the fault models are not independent of HDTO. We had better choose a fault model that is inverted using independent methods such as those inverted by seismic waveforms.

\section{Case study of a moderate dip angle seismic event: The 2008 Wenchuan earthquake}

The large Wenchuan (China) earthquake $\left(M_{\mathrm{W}} 7.9\right)$, which occurred in 12 May 2008, killed about 87000 people. The coseismic displacements at 122 GPS stations around the epicenter were detected by GPS networks operated by the China Earthquake Administration and State Bureau of Surveying and Mapping (Zhang, 2008). The magnitude of this earthquake was $M_{\mathrm{W}} 7.9$; the coseismic displacements caused by this earthquake were observed in both near and far fields. It provides us a good opportunity to study the effect of Earth's curvature and radial heterogeneity on coseismic deformations.

Many scientists have investigated the source rupture process of the 2008 Wenchuan earthquake. Ji and Hayes (2008) presented a fault model that had been inverted by teleseismic waves on the website of the U.S. Geological Survey (USGS); Zhang et al (2008) pre- sented a fault model that had been inverted from seismic wave data; Wang et al (2008) constructed a more realistic double-paliform finite-fault model inverted by teleseismic waveforms, the local geologic data and the surface rupture investigation; and so on. Among them, the fault model of Wang et al (2008) is most reliable because it is inverted by multitudinal data including GPS data. However, fault models inverted by GPS data must be avoided since we also try to compare our theoretical results with the GPS observations. Because of this reason we choose the fault model of Ji and Gavin (USGS, 2008) for this study. The dip angle of the fault plane is about 33 deg. We calculate the theoretical coseismic horizontal displacements at 122 GPS stations using SDTS and HDTO, respectively. Then we obtain the discrepancies between the two results using the following equation

$$
\bar{U}_{\text {absolute }}=\bar{U}_{\text {SDTS }}-\bar{U}_{\text {HDTO }}
$$

Therein, $\bar{U}_{\mathrm{SDTS}}$ denotes the coseismic horizontal displacements calculated by SDTS; $\bar{U}_{\text {HDTO denotes }}$ those calculated by HDTO. The difference of the two results $\bar{U}_{\text {absolute }}$ can be regarded as attributable to the effects of Earth's curvature and radical heterogeneity on coseismic displacements.

Figure 2a presents the distribution of $\bar{U}_{\text {absolute }}$ for the 2008 Wenchuan earthquake. The values of $\bar{U}_{\text {absolute }}$ are large in the near field, but they attenuate with the departure from the fault plane. The largest ones locate on both the hanging wall and footwall in directions apart from the fault plane. In contrast, those located around the extensional line of the fault plane are small but with directions headed to the fault plane. The irregular distribution of $\bar{U}_{\text {absolute }}$ at those stations near the fault plane results from numerical errors: the accuracy of the results calculated using SDTS near the fault plane is not very high because of its numerical algorithm.

Coseismic displacements in near fields are usually much larger than those in far fields. Therefore, the distribution of $\bar{U}_{\text {absolute }}$ in Figure 2a is insufficient for us to estimate the effects of Earth's curvature and radial heterogeneity. Therefore, we plot the contour maps of $\bar{U}_{\text {SDTS }}$ and $\bar{U}_{\text {HDTO }}$ in Figures $2 \mathrm{~b}$ and 2c based on uniform grids with intervals of $0.25 \mathrm{deg}$. In fact, $\bar{U}_{\mathrm{SDTS}}$ have the same distribution patterns as those of $\bar{U}_{\text {HDTO }}$ but with much smaller values, which means the effects 

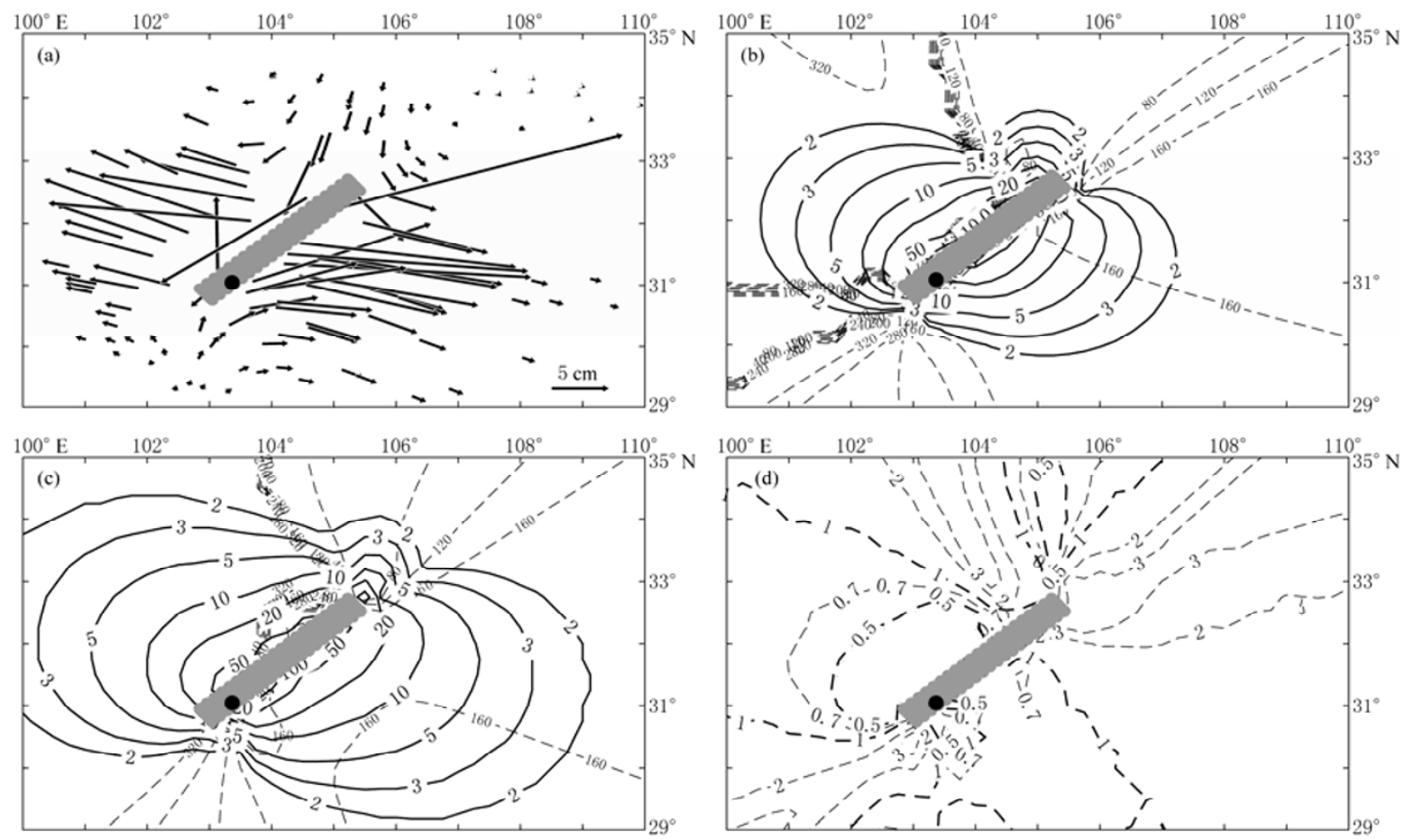

Figure 2 Differences between the theoretical results of the spherical dislocation theory (SDTS; Sun et al, 1996) and half-space dislocation theory (HDTO; Okada, 1985) for the 2008 Wenchuan earthquake. A gray area shows the fault plane. A black dot represents the epicenter. (a) Distribution of $\bar{U}_{\text {absolute }}$; (b) Contour map of results calculated using SDTS; (c) Contour map of results of HDTO. In (b) and (c), solid and dashed lines respectively give contours of lengths and directions of the displacements. The displacement unit is centimeters. Direction is measured from east in a counterclockwise rotation. (d) Contour map of $\bar{U}_{\text {relative }}$. The value of $\bar{U}_{\text {relative }}$ is dimensionless.

of Earth's curvature and radial heterogeneity are not small. To elucidate effects more clearly, we calculate the relative values $\bar{U}_{\text {relative }}$ using a simple formula as

$$
\bar{U}_{\text {relative }}=\frac{\left|\bar{U}_{\text {SDTS }}-\bar{U}_{\text {HDTO }}\right|}{\left|\bar{U}_{\text {SDTS }}\right|} \text {. }
$$

In that equation, $\bar{U}_{\text {relative }}$ is the ratio of the discrepancy between the theoretical displacements of SDTS and HDTO to the result of SDTS; it is therefore dimensionless. Figure $2 \mathrm{~d}$ presents distributions of $\bar{U}_{\text {relative }}$ for the 2008 Wenchuan earthquake. Note that we should investigate the value of $\bar{U}_{\text {relative }}$ where the value of $\bar{U}_{\text {SDTS }}$, as well as $\bar{U}_{\text {HDTO }}$, are relatively large. The big values of $\bar{U}_{\text {relative }}$ near the nodal lines of $\bar{U}_{\text {SDTS }}$ are amplified therefore meaningless. In other words, we must investigate $\bar{U}_{\text {relative }}$ in the areas west-northwest and east-southeast to the fault plane (Figure 2d). We can find that $\bar{U}_{\text {relative }}$ are larger than 0.5 in the whole study area, except for small places of the hanging wall. The effects of Earth's curvature and radial heterogeneity are about 1-2 times of the far field coseismic displacements calculated by SDTS. Ignoring the effects will cause $100 \%-200 \%$ errors on the theoretical displacements for the 2008 Wenchuan earthquake. Therefore, it is very important to replace HDTO by SDTS to invert a fault model of the 2008 Wenchuan earthquake from the coseismic displacements observed using GPS.

The value of $\bar{U}_{\text {relative }}$ in areas near the extensional line of the fault plane is 2-3 times greater than that of $\bar{U}_{\text {SDTS }}$. Such large values arise for two reasons. First, $\bar{U}_{\text {SDTS }}$ in the areas is too small (see Figure 2b), and the denominator in equation (2) amplifies the $\bar{U}_{\text {relative }}$ value. Second, the areas are near the nodal lines of the 2008 Wenchuan earthquake. Around these areas, the displacements are very sensitive. Small changes in the algorithm, as in the observation location and fault model, engender large changes in displacement. 
Finally, to investigate the discrepancies between the algorithms of SDTS and HDTO further, the root mean square (RMS) errors of the differences between the theoretical results calculated by the two dislocation theories and the observed ones are computed using the following formula

$$
\hat{\sigma}=\sqrt{\frac{\sum_{i=1}^{n}\left[\bar{U}_{\mathrm{obs}}^{i}-\bar{U}_{\mathrm{cal}}^{i}\right]^{2}}{n} .}
$$

Therein, $\bar{U}_{\text {obs }}^{i}$ denotes observations; also, $\bar{U}_{\text {cal }}^{l}$ denotes calculations, and $n$ is the number of observations. Note that $\bar{U}_{\text {obs }}^{i}$ and $\bar{U}_{\text {cal }}^{i}$ are vectors but not scalars. The RMS errors are calculated and presented in Table 1. The RMS errors of two algorithms are fundamentally equal for all 122 GPS stations. They are 509 and $507 \mathrm{~mm}$, respectively. Large discrepancies between observations and calculations are caused by the fault model inverted from teleseismic waveforms, which can reflect the average information of the fault rupture but not the detailed ones that have large effects on local deformations. Overall, it is difficult to say which algorithm is better. However, strictly regarding the RMS errors for those stations in far fields, it is apparent that SDTS interprets the observations better than HDTO does. We deduce the RMS errors according to the magnitude of the observations because displacements in a far field are much smaller than those in the near field. We use progressively smaller observations for more and more distant fields. Regarding the statistics presented in Table 1, for the far field (for those observed coseismic displacements smaller than $50 \mathrm{~mm}$ ), the RMS errors between the observation and calculation by SDTS are smaller than those by HDTO. The RMS errors of SDTS are $30 \%-50 \%$ smaller than those of HDTO in the far field. Our data results verify that SDTS works better than HDTO in the far field.

Table 1 Comparison of the RMS errors of the two calculated coseismic displacements with measured values

\begin{tabular}{ccccccrrr}
\hline Observation $/ \mathrm{mm}$ & All & $<400$ & $<100$ & $<70$ & $<50$ & $<30$ & $<10$ \\
\hline Station number & 122 & 110 & 91 & 83 & 79 & 62 & 51 \\
SDTS/mm & 509 & 44 & 18 & 14 & 12 & 7 & 5 \\
HDTO/mm & 507 & 40 & 16 & 14 & 13 & 12 & 10 \\
\hline
\end{tabular}

\section{Case study of a low dip angle seismic event: The 2004 Sumatra earthquake}

The 2004 Sumatra earthquake occurred in 26 December 2004, causing a devastating tsunami that hit coastlines across the Indian Ocean, and killing about 310000 people. The rupture began from the epicenter and expanded north-northwest, extending 1200-1300 km along the Andaman trough (Ammon et al, 2005). It was stronger than any earthquake of the prior 40 years. The coseismic displacements caused by it were observable by GPS in far fields with epicentral distance of about $6000 \mathrm{~km}$ (Fu and Sun, 2006). The earthquake provided good opportunities to study the effects of Earth's curvature and layer structure.

We choose the fault model of Ammon et al (2005) for this study. The dip angles of the fault plane are various between $12-17 \mathrm{deg}$. We calculate $\bar{U}_{\text {SDTS }}, \bar{U}_{\text {HDTO }}$ and $\bar{U}_{\text {relative }}$, as well as $\bar{U}_{\text {absolute }}$, for the 2004 Sumatra earthquake by uniform grids with 2 deg intervals around the epicenter. Figure 3 portrays complete images of the effects of Earth's curvature and stratification for the 2004 Sumatra earthquake. Similarly to the 2008 Wen- chucan earthquake, $\bar{U}_{\text {absolute }}$ are big in near field but attenuate slowly with increased epicentral distance. The directions of $\bar{U}_{\text {absolute }}$ in total depart from the fault plane. In contrast, $\bar{U}_{\text {relative }}$ are small in the near field but increase as the epicentral distance increases. Overall, $\bar{U}_{\text {relative }}$ is smaller than 1 for the 2004 Sumatra earthquake. Only in four small areas are $\bar{U}_{\text {relative }}$ larger than 1, where $\bar{U}_{\text {SDTs }}$ are small and sensitive, small changes in algorithms result in large values of $\bar{U}_{\text {relative }}$.

The effects of Earth's curvature and layer structure for the 2004 Sumatra earthquake are apparently smaller than those for the 2008 Wenchuan earthquake, which indicates that the conclusions of previous studies (Banerjee et al, 2005, 2007; Sun et al, 2009, etc) about the effects are less than normal since those investigations are all based on the 2004 Sumatra earthquake.

\section{Large effects of Earth's curvature and radial heterogeneity for moderate dip angle events}

Comparison with Figures 2 and 3 shows that the effects of Earth's curvature and radial heterogeneity for 

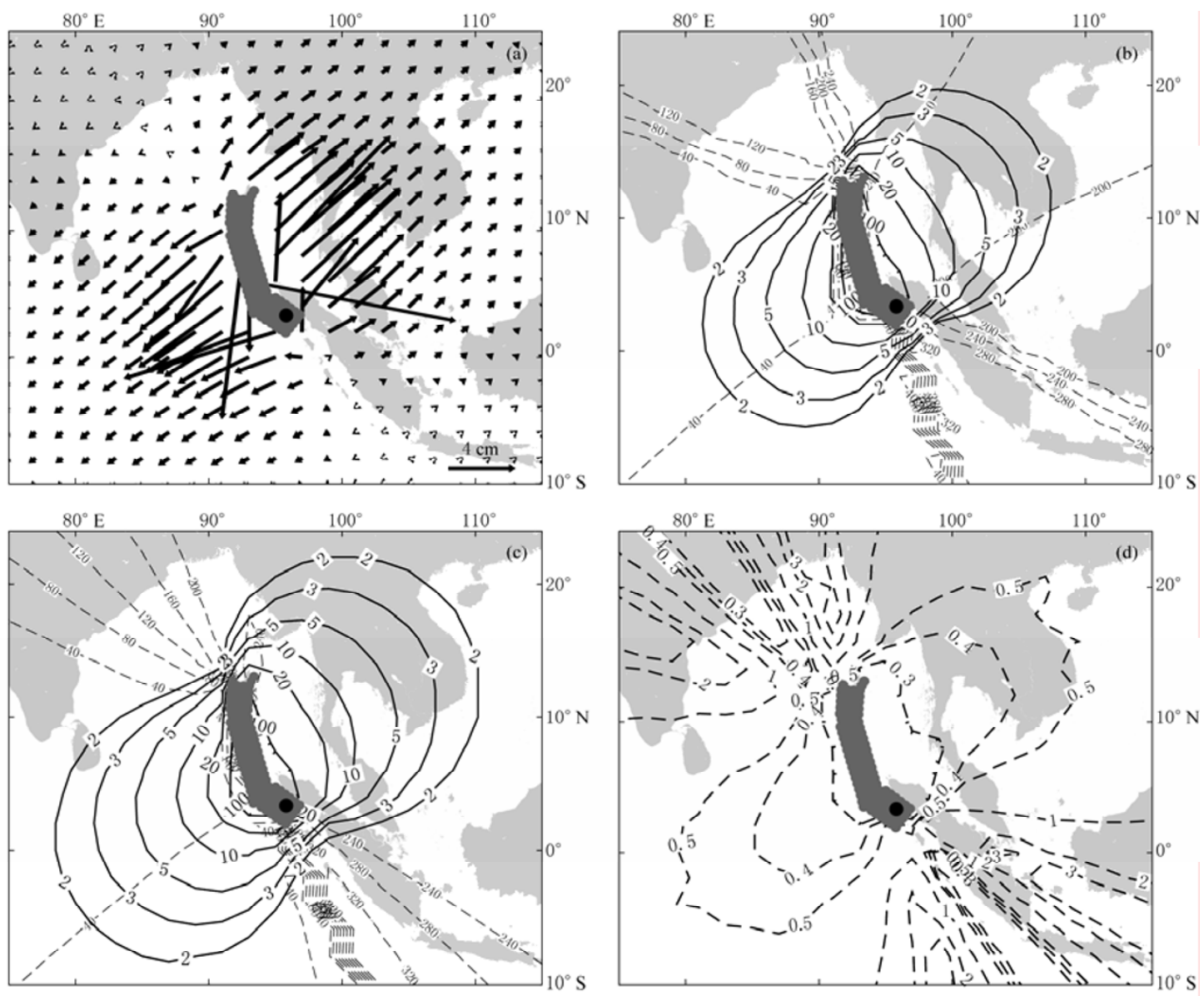

Figure 3 Distribution of $\bar{U}_{\text {absolute }}$ (a), contour maps of $\bar{U}_{\text {SDTS }}$ (b), $\bar{U}_{\text {нDTо }}$ (c) and $\bar{U}_{\text {relative }}$ (d) for the 2004 Sumatra earthquake. In Figure $3 \mathrm{~d}$, the big value of $\bar{U}_{\text {relative }}$ in areas northwest and southeast to the fault plane are meaningless due to the small values of $\bar{U}_{\text {SDTS }}$ in the same areas. Other discussions are as the same as that in Figure 2.

the 2004 Sumatra earthquake are much smaller than those for the 2008 Wenchuan earthquake. This difference results from the different dip angles of the fault planes for the two earthquakes. In another word, the effects for moderate dip angle events are larger than those for high or low dip angle events. The dip angles of the fault planes are about $33 \mathrm{deg}$ for the 2008 Wenchuan earthquake but only about 12-17 deg for the $2004 \mathrm{Su}-$ matra earthquake. That is why the effects of Earth's curvature and stratification for the 2008 Wenchuan earthquake are much greater than those for the latter one.

According to Sun and Okubo (1993), an arbitrary dislocation within a spherical symmetrical earth model can be expressed by four independent components: a vertical strike slip $u^{12}$, a vertical dip slip $u^{32}$, a vertical tensile fault $u^{22}$, and a horizontal tensile fault $u^{33}$. The excited coseismic displacements can be expressed as
(Sun et al, 2006)

$$
\begin{aligned}
& u^{\mathrm{s}}(a, \theta, \phi)=\left\{\cos \lambda\left[u^{12} \sin \delta-u^{13} \cos \delta\right]+\right. \\
& \sin \lambda\left[\frac{\left(u^{33}-u^{22}\right) \sin 2 \delta}{2}-u^{32} \cos 2 \delta\right] \frac{U d S}{a^{2}} .
\end{aligned}
$$

Therein, $u^{13}(a, \theta, \phi)=u^{32}\left(a, \theta, \phi+\frac{\pi}{2}\right), u^{\mathrm{s}}$ denotes the excited displacements caused by a shear dislocation, $a$ denotes the radius of the Earth, $\theta$ and $\phi$ are the co-latitude and longitude of the observation point, $\lambda$ denotes the slip angle of the dislocation, $\delta$ is the dip angle of the fault plane, $U$ is the magnitude of the dislocation, and $S$ is the area of dislocation.

For different dislocations, the contributions of each component are different according to the dip angle of the fault plane $\delta$ [equation (4)]. Sun and Okubo (2002) pointed out that effects of Earth's stratification are extremely large for a tensile fault $u^{22}$, even showing an 
opposite sign at the epicenter. Meanwhile the effects for other three independent components are insignificant. In another word, the differences between Green Functions of $u^{22}$ in half-space Earth model and the ones in spherical Earth model are very large. Therefore, if the dip angle of the fault plane $\delta$ is moderate, the contribution of the tensile component $u^{22}$ becomes great due to the factor $\sin 2 \delta$ in equation (4). Because of this reason $\bar{U}_{\text {absolute }}$ becomes great. That is why the effects of Earth's radial heterogeneity are very large if the dip angle $\delta$ is moderate.

\section{Discussion and conclusions}

In this study, dislocation theories of Okada (1985) and Sun et al (1996) are used to calculate coseismic displacements caused respectively by the 2008 Wenchuan earthquake and the 2004 Sumatra earthquake. Effects of Earth's curvature and stratification are investigated through discrepancies of theoretical results calculated using the two dislocation theories. Results show that the effects of Earth's curvature and stratification on coseismic deformations for the 2008 Wenchuuan earthquake are much larger than those for the 2004 Sumatra earthquake. In other words, the effects for moderate dip angle events are much greater than those for high or low dip angle events. Therefore a low dip angle event such as the 2004 Sumatra earthquake is not a good case for a topic investigating the effects of Earth's curvature and layered structure on coseismic deformations.

Our calculations show that for moderate dip angle events such as the 2008 Wenchuan earthquake, ignoring the effects of Earth's curvature and stratification will engender errors up to $100 \%-200 \%$ on far field displacements. Such large effects are much bigger than those conclusions of previous studies. Sun and Okubo (2002) claimed that the effects of the Earth's curvature and layer structure are large: as much as $25 \%$ for the effect of layer structure. However, their estimation is only an average value for special point dislocation cases. Banerjee et al $(2005,2007)$ and Sun et al (2009) studied the effects of Earth's curvature and radial heterogeneity based on the 2004 Sumatra earthquake. However, since the effects on coseismic deformations for the great earthquake are relatively small, the effects presented by those studies $(<50 \%)$ are not large enough.

Theoretical speaking, we should use spherical dislocation theory but not half space ones to explain the coseismic displacements in far filed because Earth's spherical curvature and radial heterogeneity should be considered. Our comparison with the observations verifies above conclusion since the spherical dislocation theories are better than the half-space ones for interpretation of the observed coseismic displacements in far fields (but vice versa in near fields). Therefore, we recommend the spherical dislocation theories instead the half-space ones for interpretation of the coseismic deformations in far fields or inverting a seismic fault model using far-field geodetic data, particularly for moderate dip angle seismic events.

Acknowledgments This study is supported by Grant-in-Aid for JSPS Fellows (No.20.08025) and Basic Research Foundation from Institute of Earthquake Science (No.0210240101). The authors thank two anonymous reviewers for their helpful comments and suggestions.

\section{References}

Ammon C J, Ji C, Thio H, Robinson, D, Ni S, Hjorleifsdottir V, Kanamori H, Lay T, Das S, Helmberger D, Ichinose G., Polet J and Wald D (2005). Rupture process of the 2004 Sumatra-Andaman earthquake. Science 308: 1 133-1 139.

Banerjee P, Pollitz F F and Burgmann R (2005). The size and duration of the great 2004 Sumatra-Andaman earthquake from far-field static offsets. Science 308: 1769-1 772 .

Banerjee P, Pollitz F F and Burgmann R (2007). Coseismic slip distributions of the 26 December 2004 Sumatra-Andaman and 28 March 2005 Nias earthquakes from GPS static offsets. Bull Seism Soc Amer 97(1A): S86-S102.

Dziewonski A M and Anderson D L (1981). Preliminary reference Earth model. Phys Earth Planet Inter 25(4): 297-356.

$\mathrm{Fu} \mathrm{G}$ and Sun W (2004). Effects of spatial distribution of fault slip on calculating co-seismic displacement: Case studies of the Chi-Chi earthquake $\left(M_{\mathrm{W}} 7.6\right)$ and the Kunlun earthquake $\left(M_{\mathrm{W}} 7.8\right)$. Geophys Res Lett 31: L21601.

Fu G. and Sun W (2006). Global co-seismic displacements caused by the 2004 Sumatra-Andaman earthquake $\left(M_{\mathrm{W}} 9.1\right)$. Earth Planets Space 58(2): $149-152$.

Fu G and Sun W (2008). Surface coseismic gravity changes caused by dislocations in a 3-D heterogeneous earth. Geophys J Int 172(2): 479-503.

Ji C and Hayes G (2008). Finite fault model of the May 12, $2008 \mathrm{M}_{\mathrm{W}} 7.9$ eastern Sichuan, China Earthquake. National Earthquake Information Center, United States Geological Survey. http://earthquake.usgs.gov/ earthquakes/eqinthenews/2008/us2008ryan/finite_fault.php, [2008-05-15].

Maruyama T (1964). Statical elastic dislocations in an infinite and semi-infinite medium. Bull Earthq Res Inst 42: 289-368.

Okada Y (1985) Surface deformation due to shear and tensile faults in a half-space. Bull Seism Soc Amer 75(4): 1 135-1 154.

Okubo S (1991). Potential and gravity changes raised by point dislocations. Geophys J Int 105(3): 573-586.

Okubo S (1992). Potential and gravity changes due to shear and tensile faults in a half-space. J Geophys Res 97(B5): 7 137-7 144.

Pollitz F F (2003). Post-seismic relaxation theory on a laterally heterogeneous viscoelastic model. Geophys J Int 155(1): 57-78.

Sabadini R and Vermeersen L L A (1997). Influence of lithospheric and mantle stratification on global post-seismic deformation. Geophys Res Lett 24(16): $2075-2078$.

Steketee J A (1958). On Volterra's dislocations in a semi-infinite elastic me- 
dium. Can J Phys 36(2): 192-205.

Sun W and Okubo S (1993). Surface potential and gravity changes due to internal dislocations in a spherical earth (I): Theory for a point dislocation. Geophys J Int 114(3): 569-592.

Sun W and Okubo S (1998). Surface potential and gravity changes due to internal dislocations in a spherical earth ( II ): Application to a finite fault. Geophys J Int 132(1): 79-88.

Sun W and Okubo S (2002). Effect of Earth's spherical curvature and radial heterogeneity in dislocation studies: For a point dislocation. Geophys Res Lett 29(12), doi: 10.1029/2001GL014497.

Sun W, Okubo S and Vanicek P (1996). Global displacement caused by dislocations in a realistic Earth model. J Geophys Res 101(B4): 8 561-8 577.

Sun W, Okubo S and Fu G (2006). Green's functions of co-seismic strain changes and investigation of effect of Earth's spherical curvature and radial heterogeneity. Geophys J Int 167(3): 1273-1 291.

Sun W, Okubo S, Fu G and Araya A (2009). General formulations of global coseismic deformations caused by an arbitrary dislocation in a spherically symmetric Earth model: Applicable to deformed Earth surface and space-fixed point. Geophys J Int 177(3): 817-833.

Tanaka Y, Okuno J and Okubo S (2006). A new method for the computation of global viscoelastic post-seismic deformation in a realistic earth model (I): Vertical displacement and gravity variation. Geophys J Int 164(2): 273-289.

Wang W, Zhao L, Li J and Yao Z (2008). Rupture process of the $M_{\mathrm{S}} 8.0$ Wenchuan Earthquake of Sichuan, China. Chinese J Geophys 51(5): 1403 1410 (in Chinese).

Zhang P (2008). Coseismic displacements of the 2008 Wenchuan earthquake $\left(M_{\mathrm{S}} 8.0\right)$ detected by GPS. Science in China (Series D) 38(10): 1 195-1 206 (in Chinese).

Zhang R, Feng W, Xiu L, Zhou C and Chen Y (2008). Rupture process of the 2008 Wenchuan earthquake. Science in China (Series D) 38(10): 1 1861194 (in Chinese). 